\title{
High Frequency Operation of Series-Connected IGCTs for Resonant Converters
}

G. Ulissi, U. Vemulapati, T. Stiasny, et al.

This material is posted here with permission of the IEEE. Such permission of the IEEE does not in any way imply IEEE endorsement of any of EPFL's products or services. Internal or personal use of this material is permitted. However, permission to reprint / republish this material for advertising or promotional purposes or for creating new collective works for resale or redistribution must be obtained from the IEEE by writing to pubs-permissions@ieee. org. By choosing to view this document, you agree to all provisions of the copyright laws protecting it. 


\title{
High Frequency Operation of Series-Connected IGCTs for Resonant Converters
}

\author{
Gabriele Ulissi, Graduate Student Member, IEEE, Umamaheswara Reddy Vemulapati, Member, IEEE, \\ Thomas Stiasny, and Drazen Dujic, Senior Member, IEEE
}

\begin{abstract}
Series connected IGCTs find application in hard-switched commercial power converters at the MW power levels. Expanding the application of these devices to medium voltage dc-dc resonant converters is of great interest due to the IGCT's low conduction losses. This requires a deeper understanding of the device's behaviour in series connection and under switching conditions different than those in hard switched converters. In particular, the voltage sharing between series connected IGCTs must be achieved with significantly reduced turn-off currents, typical for resonant converters. Relying on a versatile IGCT test setup, this paper explores the operational performances of series connected IGCT operation at very low turn-off current and with snubber capacitance in the range of a few tens of $\mathrm{nF}$. The results presented in the paper are obtained from commercially available $4.5 \mathrm{kV} 68 \mathrm{~mm}$ reverse conducting IGCTs (with standard irradiation), as well as two customized engineering samples (high irradiated variants), optimized on their technology curve for high switching frequencies. The IGCTs are successfully operated in series connection at $5 \mathrm{kHz}$, with device voltage being effectively shared in resonant converter operation.
\end{abstract}

\section{INTRODUCTION}

The ongoing drive for ever-increasing efficiency in transport and conversion of electrical energy has lead to the desire to increase the voltage of dc systems from the low-voltage (LV) to the medium-voltage (MV) level in a number of applications[1]-[4]. Compared to their ac counterparts, direct current (DC) networks offer higher efficiency, no need for reactive power compensation, and no need for grid synchronisation. Additionally, simple integration of renewables, energy storage systems and DC loads such as fast charging stations and data centres make medium-voltage direct current (MVdc) grids more flexible and appealing for both onshore and offshore use, driving ongoing academic and industrial efforts [5]-[8].

For several years now, bidirectional isolated power converters interfacing dc buses of different voltages at the MV level as shown in Fig. 1, have been identified as a key enabling technology for the development of MVdc systems [9]-[11]. A number of different solutions have been proposed, with dual active bridge (DAB) and LLC-series resonant converter (SRC)

Gabriele Ulissi and Drazen Dujic are with the Power Electronics Laboratory, Ecole Polytechnique Fédérale de Lausanne (EPFL), 1015 Lausanne, Switzerland (e-mail: gabriele.ulissi@epfl.ch; drazen.dujic@epfl.ch). Umamaheswara Vemulapati and Thomas Stiasny are with Hitachi ABB Power Grids, Semiconductors, 5600, Lenzburg, Switzerland (e-mail: umamaheswara.vemulapati@hitachipowergrids.com; thomas.stiasny@hitachi-powergrids.com).

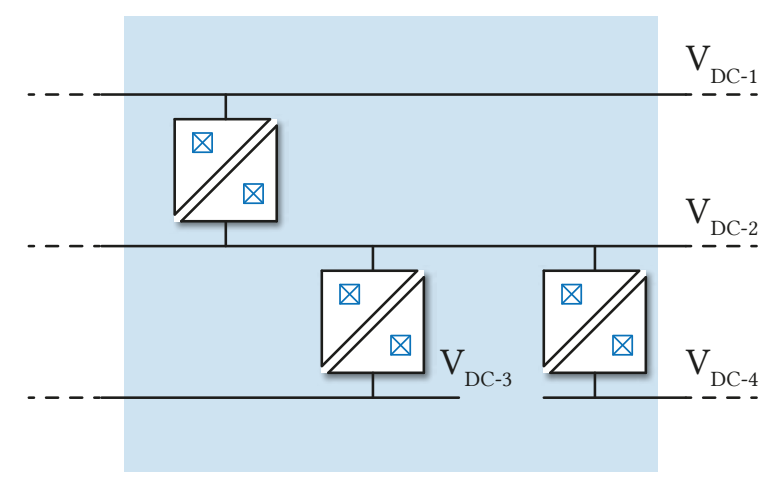

Fig. 1: DC transformers in medium-voltage direct current systems provide interfaces between buses of different voltage level and isolation.

being the most popular topologies and with the DAB gaining significant traction particularly in the marine and offshore wind farm sectors [12]-[15]. Nevertheless, DAB based converters require closed loop control [16], which does not striclty correspond to the definition of dc transformer (DCX). The SRC on the other hand, can provide bidirectional energy flow while in open loop operation thanks to its stiff voltage transfer characteristic [17]. Additionally, by increasing the semiconductor switching frequency, the volume and mass of both the medium-frequency (MF) transformer and resonant tank capacitors can be significantly reduced with positive repercussions on the DCX's power density, cost, and size. The desire for increasingly high frequency operation has brought the insulated-gate bipolar transistor (IGBT) and SiC MOSFET to be the most commonly considered devices for DCX applications [18], [19]. The first provides ruggedness, ease of drive, and wide availability [12]-[15], while the second allows for increased switching frequencies and reduced losses.

In spite of the popularity of these devices, over the past thirty years the integrated gate-commutated thyristor (IGCT) has established itself as a relevant competitor in the field of power conversion at the MV level in multi-MW systems [20], [21]. The device has proven to be an effective alternative to the popular IGBT as the IGCT offers increased reliability, larger safe operating area (SOA), better utilisation of the silicon wafer area in press-packed devices and therefore better thermal contact with the cooling surface. Additionally, its thyristor 
based nature guarantees significantly lower conduction losses than an IGBT of similar ratings [22]-[28]. On the other hand historically, the IGCT has been optimised for operation in hard switched conditions (several $\mathrm{kA}, \mathrm{kV}$ ) which are typical for high power inverters. In these conditions the device's turn-off energy is significant. Due to the resulting relatively high switching losses, the switching frequency of the device normally does not exceed $1 \mathrm{kHz}$.

Nevertheless, recent research has shown that the limitation on the IGCT switching frequency can be effectively sidestepped by capitalising on the advantageous switching conditions provided by SRC based DCX [29], [30]. In particular, topologies such as the LLC-SRC in subresonant operation, shown in Fig. 4 can provide truly optimal operating conditions for IGCTs. In such a topology and operating mode, the power transfer between primary and secondary takes place during the resonant current pulse, which is spontaneously ended after a time interval determined by the resonant frequency of the converter's tank. This leads to a large resonant current peak, which is efficiently handled thanks to the IGCT's low on-state losses. The IGCT turn-off, normally a significant source of losses, is performed at extremely low current levels, independent of load condition and determined by the magnetising inductance of the transformer. In these conditions turn-on losses are also negligible due to the zero voltage switching (ZVS) or zero current switching (ZCS) conditions at turn-on, depending on the converter load. Finally, the clamp circuit conventionally needed to protect the IGCT antiparallel diode can be entirely omitted thanks to the current rate of increase being naturally limited by the resonant tank. Operation of IGCTs in these favourable conditions has been shown to be possible in the range of several $\mathrm{kHz}$, providing a range of benefits related to the reduced footprint, weight and cost of IGCT-based DCX [31].

To truly support the adoption of MVdc power systems on a large scale through the use of LLC-SRC based DCX, the

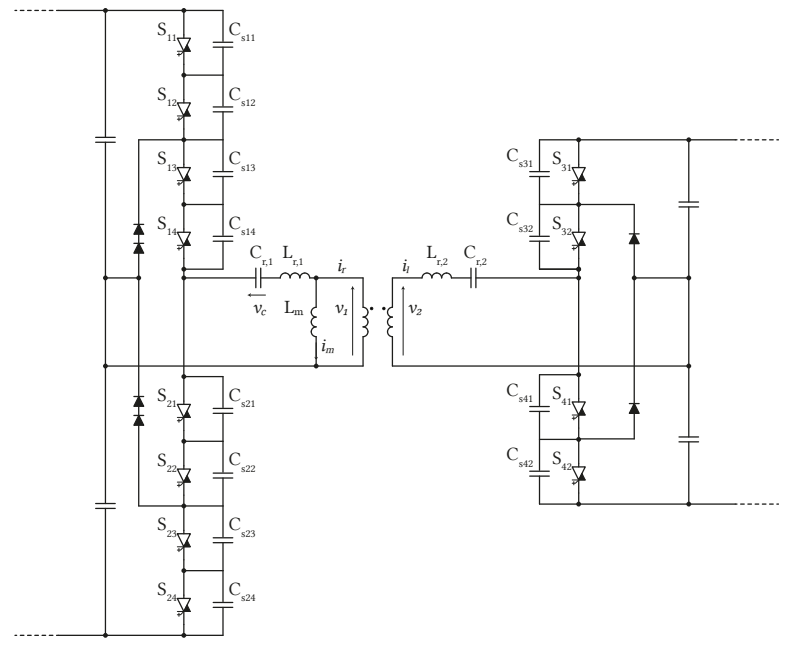

Fig. 2: IGCT-based LLC-SRC for bulk power transfer. NPC topology is employed on both high $(10 \mathrm{kV})$ and low $(5 \mathrm{kV})$ side. scalability of such a device to increased voltage levels needs to be demonstrated. This paper investigates the feasibility of such voltage scaling through the series connection of $4.5 \mathrm{kV}$ RC-IGCT devices in LLC-SRC DCX. In particular, the paper explored the ability of series connected IGCTs to effectively share voltage at turn-off under the very low current turn-off that takes place in the LLC-SRC topology. Through thorough experimental characterization, snubber capacitor values are evaluated and compared directly, in terms of their impact on the voltage sharing during switching transitions. Finally, the paper explores the performance increase offered by the use of reverse conducting IGCT (RC-IGCT) engineering samples with increased irradiation levels exhibiting reduced values of turn-off energy at the expense on increased conduction loss. Through the use of these devices, the IGCTs are operated for the first time in series connection at a frequency of $5 \mathrm{kHz}$.

The contributions of this paper are: $i)$ the analysis of voltage switching transition of commercially available seriesconnected IGCTs (with standard irradiation) and customized engineering samples (with higher irradiation) under low current switching conditions; ii) the characterization of the influence of small snubber capacitance values on switching performance at high switching frequencies; iii) the reporting for the first time of series connected IGCT operation at the record-high switching frequency of $5 \mathrm{kHz}$.

This paper is organised as follows: Section II discusses the series connection of IGCTs in LLC-SRC and the challenges arising from low current turn-off, Section III provides a description of the semiconductors employed for the characterisation of low current turn-off and the setup used to test them, Section IV provides test results and their analysis for different levels of turn-off current, snubber capacitance and IGCT devices, and finally Section V demonstrated for the first time series connected IGCT operation at a frequency of $5 \mathrm{kHz}$ thanks to the advantageous soft-switching condition and demonstrating the feasibility of DCX employing MF transformer and IGCTs.

\section{IGCT SERIES CONNECTION IN LLC-SRC}

Differently from the IGBT, which can benefit from active voltage balancing, the dynamic and static voltage sharing between series connected IGCTs is guaranteed by snubbers and balancing resistors, respectively, such as those shown in Fig. 3. This is because the turn-off process of an IGCT is quite similar to that of a traditional gate turn-off thyristor (GTO): the anode current must be commutated from the cathode to the gate through the application of negative voltage at the gatecathode junction. This sweeps charge carriers from the junction which undergoes reverse recovery and becomes reverse biased. While this process is under the control of the gate driver unit, the voltage increase at the device's terminals only takes place during the sweep-out of the n-base, which only takes place later in the turn-off process and is affected only by the characteristics of the device, and not by the gate driver unit action. The series connection of RC-IGCTs in hard switched applications and the relative snubber sizing is well documented in academic publications and employed in industrial products 


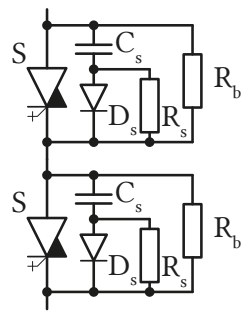

(a)

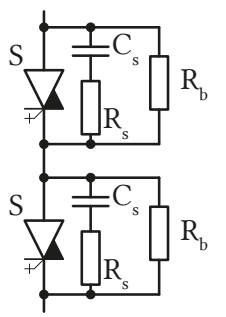

(b)

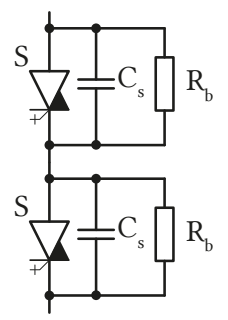

(c)
Fig. 3: Series connected RC-IGCTs with (a) RCD, (b) RC and (c) $\mathrm{C}$ parallel connected snubber for dynamic voltage balancing.

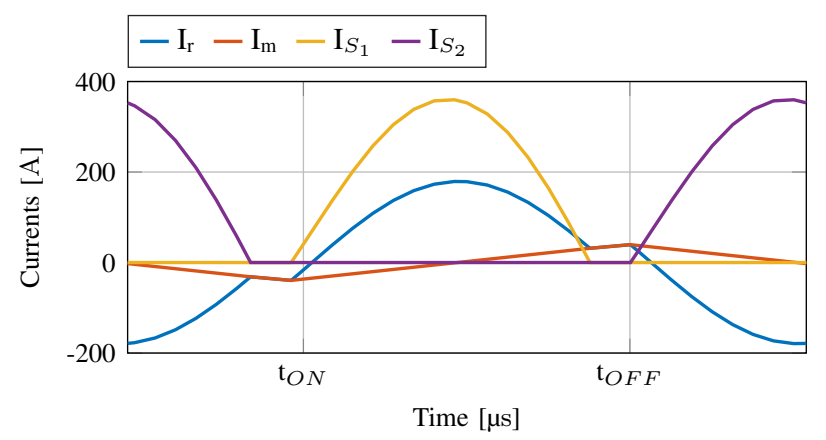

Fig. 4: IGCT turn-on in the LLC-SRC is performed in ZVS condition thanks to the current conducted by the device's antiparallel diode. The turn-off take place in low current conditions.

[32]-[36]. Contrarily, series connection of RC-IGCTs in soft switched applications has rarely been investigated and poses some challenges. The principles employed are the same as in hard-switched applications. However, soft-switched resonant converters allow for significant simplification of the dynamic voltage balancing snubbers. Concerning the static voltage balancing resistors ( $R_{b}$ in the figure), these achieve their goal by conducting a larger current than the leakage current of the RC-IGCTs and their antiparallel diodes while the IGCTs are $O F F$. Reference [37] provides a sizing rule in the form of

$$
\frac{\frac{V_{d c}}{n}-\frac{\Delta V}{n-1}}{R_{b}-\Delta R_{b}}+I_{l 0, \max } \sqrt{\frac{\frac{V_{d c}}{n}-\frac{\Delta V}{n-1}}{V_{I G C T, 0}}}=\frac{\frac{V_{d c}}{n}+\Delta V}{R_{b}+\Delta R_{b}},
$$

where $n$ is the number of series connected devices, $I_{l 0, \max }$ is the maximum leakage current of the devices at voltage $V_{I G C T, 0}, R_{b}$ and $\Delta R_{b}$ are the values of the balancing resistor and its tolerance, and $\Delta V$ is the maximum voltage deviation between the series connected devices. The equation can be solved numerically to calculate the required value of balancing resistors based on the acceptable level of voltage deviation, which is up to the designer.

On the other hand, the sizing of dynamic voltage sharing snubbers of the series connected RC-IGCTs is affected both by the maximum acceptable dynamic voltage imbalance, and by the expected turn-off current level. In a conventional LLCSRC converter, the transmission of power from the primary to secondary side takes place during the resonant pulse, the duration of which is determined by the resonant frequency of the converter's tank. The resonant current pulse and its relatively slow $d i / d t$ rise are what allows the turn-on of devices in the LLC-SRC topology to take place in ZVS or quasi-ZVS conditions, as seen in Fig. 4. This is very beneficial as turn-on losses in these conditions are negligible as long as the condition on the dead-time

$$
t_{d, o f f}<t_{D T}<t_{d, o f f}+t_{D, c n d}-t_{d, o n}
$$

is verified, as discussed in [31], where $t_{d, o f f}$ is the gate driver turn-off delay, $t_{d, \text { on }}$ is the turn-on delay, $t_{D T}$ is the dead time (usually around $10 \mu \mathrm{s}$ to $12 \mu \mathrm{s}$ ), and $t_{D, \text { cnd }}$ is the GCT antiparallel diode conduction time.

After the resonant pulse is spontaneously extinguished, the only remaining current in the resonant tank is the transformer magnetising current. At turn-off, the conducting devices only need to interrupt this magnetising current, the peak of which can be defined during the converter design through the magnetising inductance of the transformer. Therefore, while the conduction losses of the topology are determined by the power and voltage level, the turn-off losses are linear with the switching frequency. For this reason, in a medium-frequency dc transformer application, IGCT turn-off losses constitute the bulk of the converter losses [31]. Therefore, to allow mediumfrequency operation of the topology, the IGCT turn-off current (that is to say the peak transformer magnetising current) must be brought to a very low level, which is beneficial in terms of reducing turn-off losses and allowing for frequency increase in order to reduce the size of the MFT in the topology.

To evaluate the peak dynamic voltage difference between two series connected devices, reference [37] has proposed

$$
\Delta V_{d y n}=\frac{V_{d c}}{2} \frac{\Delta C_{s}}{2 C_{s}+\Delta C_{s}}
$$

for hard switched applications. This is the case as the IGCT output capacitance, typically in the range $0.8 \mathrm{nF}$ to $1 \mathrm{nF}$ (for $68 \mathrm{~mm}$ RC-IGCTs), can be neglected with respect to that of the snubbers. In hard switched applications, the IGCT turnoff current can be in the range of several $\mathrm{kA}$ with examples in literature of snubber capacitors in the range of $200 \mathrm{nF}$ to $1 \mu \mathrm{F}$. The voltage rise time can therefore be approximated to be equal to the time necessary for the turn-off current to charge the IGCT snubber capacitance. The switching period in hard switching application is usually above $1 \mathrm{~ms}$. Therefore, even with large snubbers, the duration of the dead time during which the voltage transition must be completed is small with respect to the switching period.

The low current turn-off in the LLC-SRC poses a more significant challenge in terms of dynamic voltage balancing. The sum of the gate driver delay $t_{\text {delay }}$ and the voltage rise time $t_{\text {rise }}$ must be lower that the selected dead-time $t_{D T}$ to ensure a safe turn-off. While a low turn-off current has a relatively small impact on the gate driver delay $t_{\text {delay }}$, the duration of the voltage rise is significantly extended by a reduction in turn-off current. An increased voltage rise time due to slow snubber capacitance charging is incompatible with the desired increase in switching frequency that can be 
achieved with a low turn-off current. The need to increase the dead-time to ensure safe commutation works against the desire to reduce the switching period, as these values of $15 \mu$ s (inherited from [31]) and $200 \mu \mathrm{s}$, respectively are in a comparable range.

To achieve increased switching frequencies, the snubber design must be such that the IGCT transitions are as fast as possible, allowing for a reduction of the dead-time. Therefore, a reduction in snubber capacitance size is required for an increase in switching frequency to be possible. Still under the assumption that the IGCT output capacitance is negligible, a sizing rule is established in this work as

$$
C_{\text {max }}=\frac{i_{o f f} n t_{D T}}{4 V_{d c}},
$$

where $i_{\text {off } f}$ is the IGCT turn-off current, $t_{D T}$ is the deadtime, $V_{d c}$ is the dc bus voltage and $n$ is the number of series connected IGCTs per position. As an example, for two series connected devices with a $5 \mathrm{kV}$ dc link voltage, $50 \mathrm{~A}$ turnoff current and a relatively long dead-time of $15 \mu$ s (values similar to those in [31]), this results in a maximum snubber capacitance of $75 \mathrm{nF}$.

Nevertheless, it should be reiterated that operation in a soft switched topology also brings very significant advantages. In particular, almost independently from the load condition, the turn-on of the IGCTs is performed in ZVS or quasi-ZVS conditions. A low voltage turn-on means there is no danger linked to the discharge of the snubber capacitance into the device as it turns on, and therefore, in principle, no need for the typically employed snubber resistor shown in Fig $3 \mathrm{~b}$. This makes the turn-on process non-critical and allows the use of purely capacitive snubbers in soft-switched topologies with significant advantages in terms of snubber physical size, cost and efficiency. Additionally, the current rate of increase at the time of IGCT turn-on is limited by the resonant tank inductive elements to a few $\frac{\mathrm{A}}{\mathrm{us}}$. This eliminates the risk of high di/dt reverse recovery of the GCT antiparallel diode and therefore the need for di/dt clamp circuitry. These two factors significantly simplify the converter structure, as already seen in Fig. 2.

Finally, it has been demonstrated in [31] that a turn-off currents as low as $50 \mathrm{~A}$ result in effective IGCT turn-off for a single device at the $2.5 \mathrm{kV}$ level. Based on this result, the remainder of this paper focuses on low current turn-off characterisation with turn-off current values of $50 \mathrm{~A}, 75 \mathrm{~A}$ and $110 \mathrm{~A}$. Snubber capacitance values of $40 \mathrm{nF}, 70 \mathrm{nF}$, and $100 \mathrm{nF}$ are selected corresponding to estimated $t_{D T}$ values included between $8 \mu$ s and $9.5 \mu \mathrm{s}$, which is sufficiently brief. In addition to voltage sharing, device losses are also calculated as a function of turn-off current and snubber capacitance values, as the IGCT turn-off is critical both in terms of device voltage sharing and overall losses.

\section{IGCT DeVices AND TEst Setup}

The IGCT devices under test are $68 \mathrm{~mm}, 4.5 \mathrm{kV}$ RC-IGCTs. The RC-IGCT wafer is shown in Fig. 5a, in which the IGCT and anti-parallel diode are integrated monolithically in one wafer. The RC-IGCT solution provides compactness by

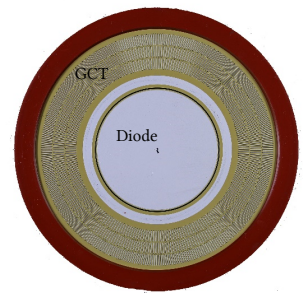

(a)

(c)

(b)
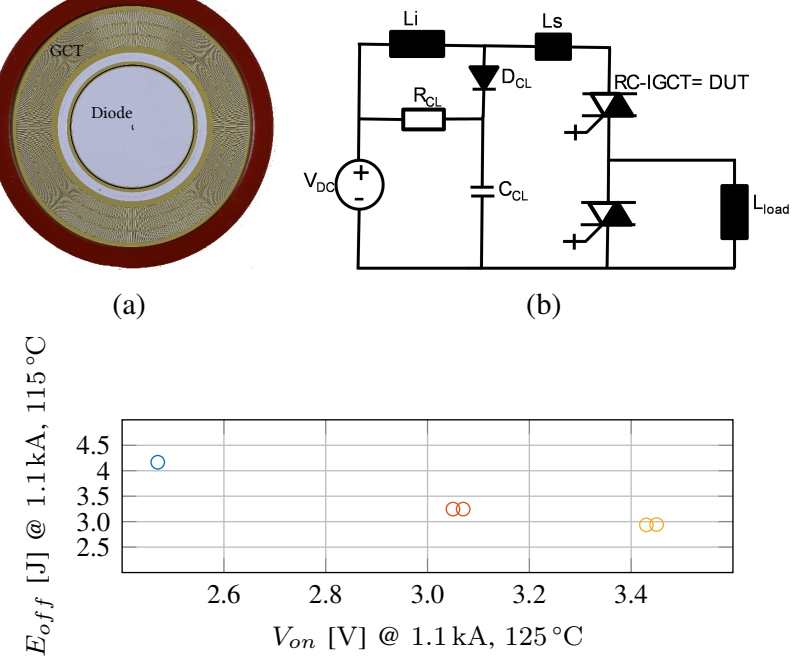

Fig. 5: (a) $68 \mathrm{~mm}, 4.5 \mathrm{kV}$ reverse conducting IGCT wafer; (b) half bridge test circuit used for hard-switched turn-off measurements; (c) turn-off energy as a function of on-state voltage as tested in setup (b) under hard switched conditions for: $\bigcirc$ standard devices, $\bigcirc$ irradiated $A$, and $\bigcirc$ irradiated $B$. The setup parameters are: $L_{i}=7.4 \mu \mathrm{H}, C_{c l}=1 \mu \mathrm{F}$, $R_{c l}=1.25 \Omega$ and $L_{s}=600 \mathrm{nH}$.

reducing the parts count at system level thereby improved reliability. Today however, IGCTs are optimized normally for state-of-the-art two to five level inverters, which are operating at relatively low switching frequencies in the range of few hundreds of $\mathrm{Hz}$ under hard switched conditions. In these applications turn-off occurs at relatively high currents compared to resonant converters, where the operating switching frequencies are in the range of few $\mathrm{kHz}$ under soft switched conditions with turn-off occurring at relatively low currents.

In order to increase the switching frequency of the commercially available $68 \mathrm{~mm}, 4.5 \mathrm{kV}$ RC-IGCTs to be used in resonant converters, two unique engineering samples are manufactured and tested. These devices were subjected to higher electron irradiation compared to commercial devices. Two design variants with $55 \%$ and $95 \%$ higher electron irradiation are referred to as Irradiated $A$ and Irradiated $B$, respectively, and are compared to the commercial devices with the standard electron irradiation. The technology trade-off of these three designs (RC-IGCTs with standard irradiation, Irradiation A and Irradiation B) are compared under hardswitched conditions (i.e. at high turn-off current and high dc-link voltage) using classical half bridge test configuration with clamp circuit as shown in Fig. 5b. As shown in Fig. 5c, the increased irradiation reduces the turn-off energy losses by $22 \%$ and $29 \%$ in Irradiated $A$ and Irradiated B, respectively, when compared to standard irradiated devices. However, the on-state voltage increases by $23 \%$ and $39 \%$ in Irradiated $A$ and Irradiated $B$, respectively. The improvement in switching performance with higher irradiation (Irradiated $B$ ) has a 
larger trade-off on the on-state under hard-switched conditions. The performance of these three designs is tested under softswitched conditions (at low turn-off currents) using the test setup shown in Fig. 6b and the results are presented in Section IV.

To characterize the performances and verify the design rules for series connection of IGCTs in low current turnoff conditions, a flexible test setup shown in Fig. 6a, is extended expanding upon what was presented in [30]. This test setup allows for DP tests and resonant operation tests of both individual and series connected IGCTs. The configuration of the test setup is shown in Fig. $6 \mathrm{~b}$ and the value of the employed components is listed in Table I.

The objectives of the test are to characterise three crucial parameters, as a function of the turn-off current and snubber capacitance. The first is the voltage rise time. This, together with the IGCT turn-off delay provide the minimum value of the converter's dead-time. Considering the low values of the turn-off current, the duration of the voltage rise is expected to be increased compared to hard switched conditions. The second, is the turn-off energy has to be computed. This is of crucial importance if the switching frequency of the converter is to be increased, as turn-off losses are the most relevant part of total converter losses. Thanks to the measurement of terminal voltage and current in each of the devices under test (DUTs), as displayed in Fig. 6, the switching energy can be calculated as the integral over time of the power dissipated in the device. Finally, the difference in voltage between the two DUTs is calculated, as the use of small snubbers increases the risk of dynamic voltage unbalance. An example of a typical test result in this configuration is shown in Fig. 6c

\section{IGCT LOW CURRENT TURN-OFF}

All three RC-IGCTs mentioned in Section III are methodically tested and their turn-off is characterised with varying levels of turn-off current and snubber components. The same commercial gate driver units are employed in all tests, and only the GCTs are replaced.

\section{A. Commercial RC-IGCTS}

Figs. 7 and 8 display the measured electrical quantities of the test setup during turn-off with $5 \Omega$ and $0 \Omega$ snubber resistance, respectively. Both figures display on the left side results for $S_{1}$ and on the right side results for $S_{2}$. The values of turn-off current used are, from top to bottom, $55 \mathrm{~A}, 75 \mathrm{~A}$ and 110 A. Small discrepancies in the values of turn-off current are due to sensing with high-current $3 \mathrm{kA}$ probes, which are the only ones available during measurements.

To summarise the results in a clear and concise fashion, Fig. 9 displays the values of the voltage rise times, turn-off energies and maximum voltage difference between the DUTs

TABLE I: Values of passive component of the test setup.

\begin{tabular}{cccccccc}
\hline \hline$C_{d c}$ & $L_{c l}$ & $R_{c l}$ & $C_{c l}$ & $C_{s}$ & $R_{s}$ & $R_{b}$ & $L_{m}$ \\
\hline $1.3 \mathrm{mF}$ & $18 \mu \mathrm{H}$ & $2 \Omega$ & $7.4 \mu \mathrm{F}$ & $40 \mathrm{nF}, 70 \mathrm{nF}, 100 \mathrm{nF}$ & $0 \Omega, 5 \Omega$ & $10 \mathrm{k} \Omega$ & $6.3 \mathrm{mH}$ \\
\hline
\end{tabular}

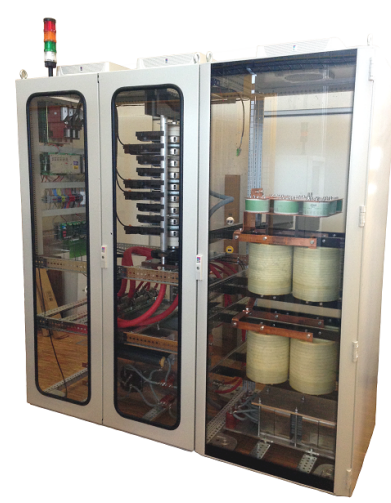

(a)

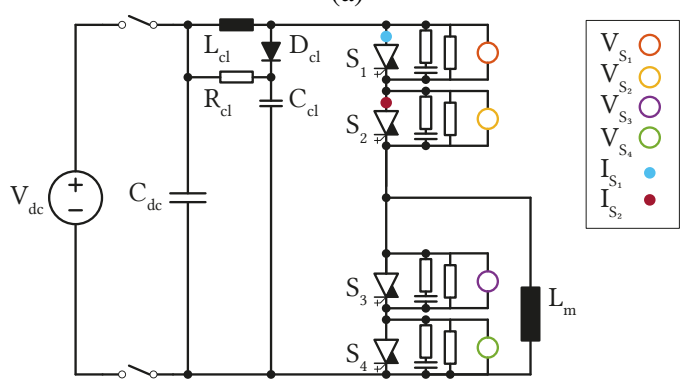

(b)
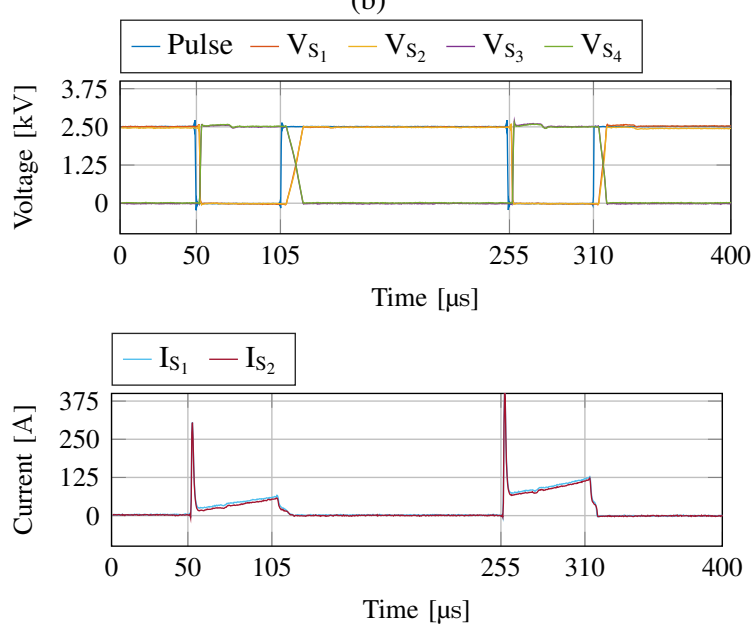

(c)

Fig. 6: (a) test setup [31] and (b) its configuration for double pulse (DP) testing, with two RC-IGCTs per position. Each GCT is provided with an RC snubber for dynamic voltage sharing, and a balancing resistor for static voltage sharing. (c) the turn-off current level at the end of each pulse is determined by the pulse duration, which is of $55 \mu \mathrm{s}$ in the test results.

during the turn-off process. Based on the figure, two expected effects can be observed:

- A larger snubber capacitance (while still very low) results in slower voltage rise, but better voltage sharing and lower turn-off energy.

- A larger turn-off current results in faster voltage rise, higher turn-off energy and larger voltage imbalance between series connected devices.

There are multiple important observations that are relevant 


\section{IEEE TRANSACTIONS ON POWER ELECTRONICS}

to series connected LLC-SRC operation. First and foremost, comparing Fig. 7 and Fig. 8, one can see that the main difference that is observable is the slight oscillations in current before turn-off that are present in the case of $R_{s}=0 \Omega$. This is not due to the turn-off process itself, but rather the turn-on of the device, which takes place in hard-switched conditions, with voltage present on the devices' terminals. Because if this hard turn-on, the snubber capacitance quickly discharges in the device upon turn-on, causing large current oscillations that are damped over time. A longer duration of the $O N$ time for $S_{1}$ and $S_{2}$ would have allowed further damping of these oscillations, but as no larger value was available for $L_{m}$, it was not possible to increase the $O N$ pulse duration and maintain the same levels of turn-off current. It is therefore important to note that the presence of snubber resistance does not affect lowcurrent turn-off of the device. Second, the maximum voltage difference between the series connected devices never exceeds $300 \mathrm{~V}$, with this value only being reached for the very small snubber capacitance of $40 \mathrm{nF}$ and the largest considered turnoff current of $100 \mathrm{~A}$. This is due to higher turn-off current and the smallest snubber capcitance resulting in higher $\frac{d v}{d t}$ and therefore exacerbating any asymmetry in switchign delay or gate driver action. All other combinations of snubber capacitance and turnoff current result in smaller peak dynamic

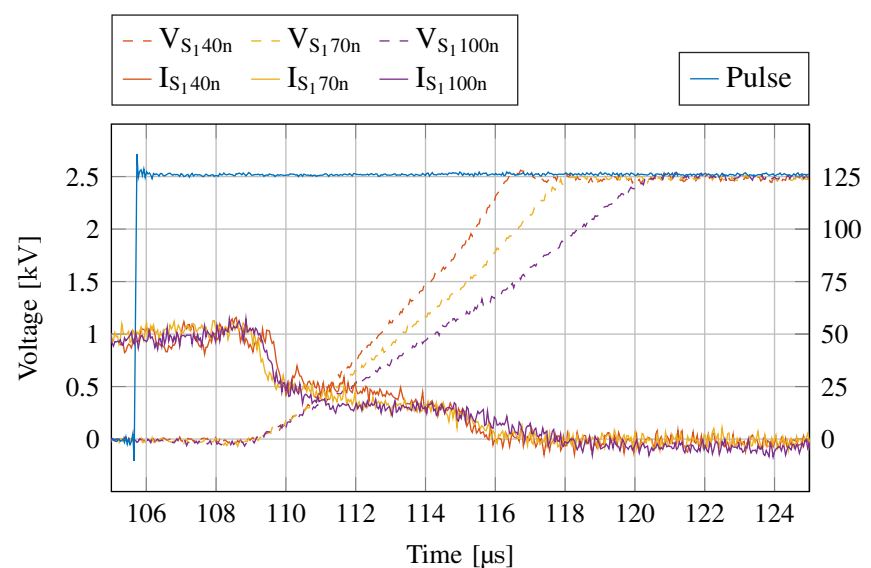

(a)

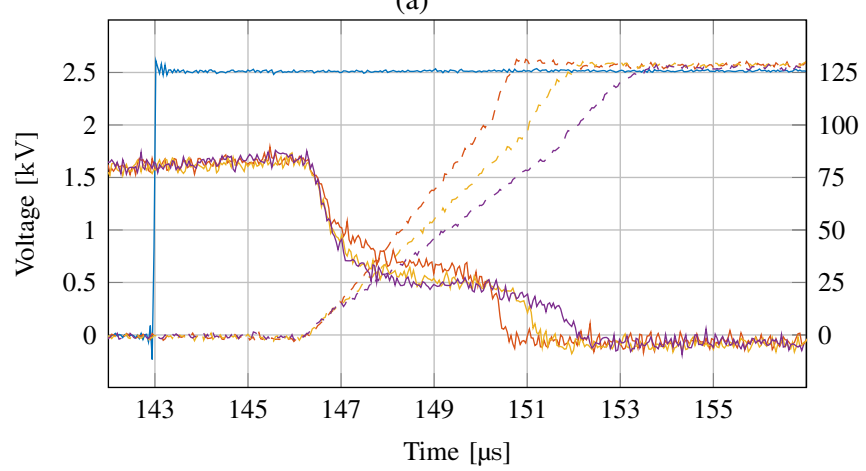

(c)

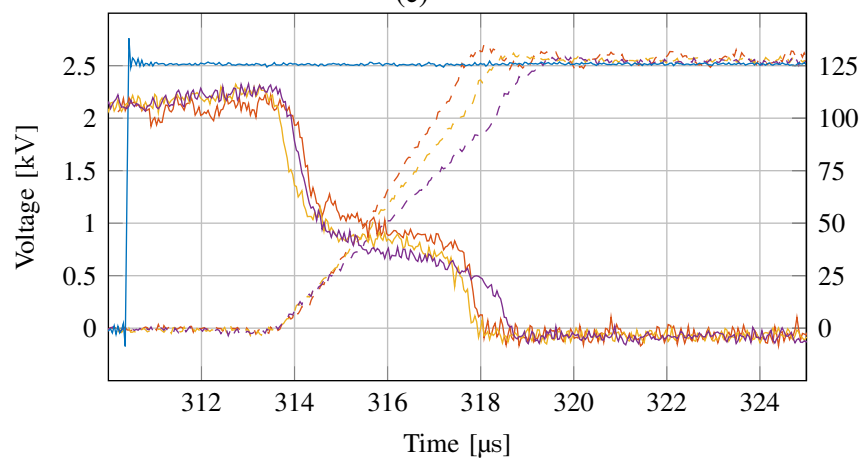

(e)

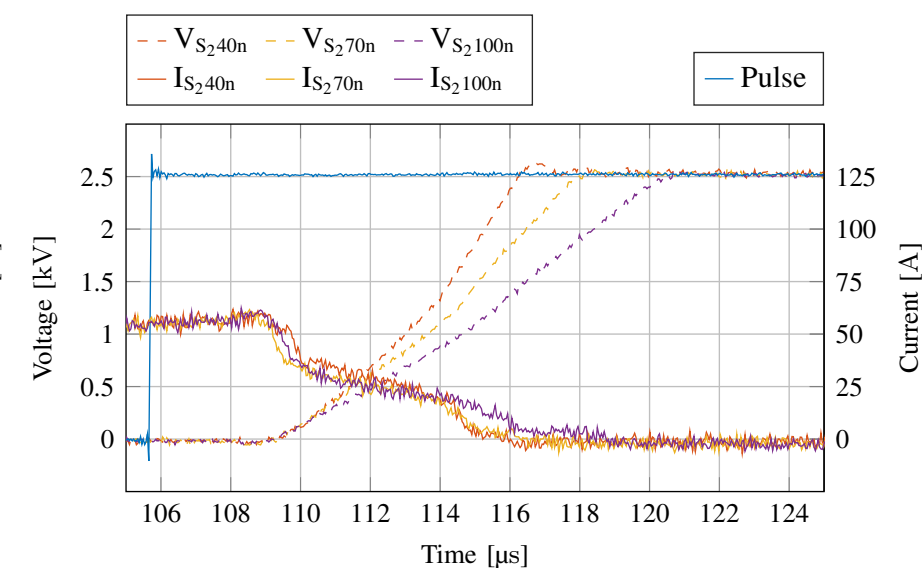

(b)

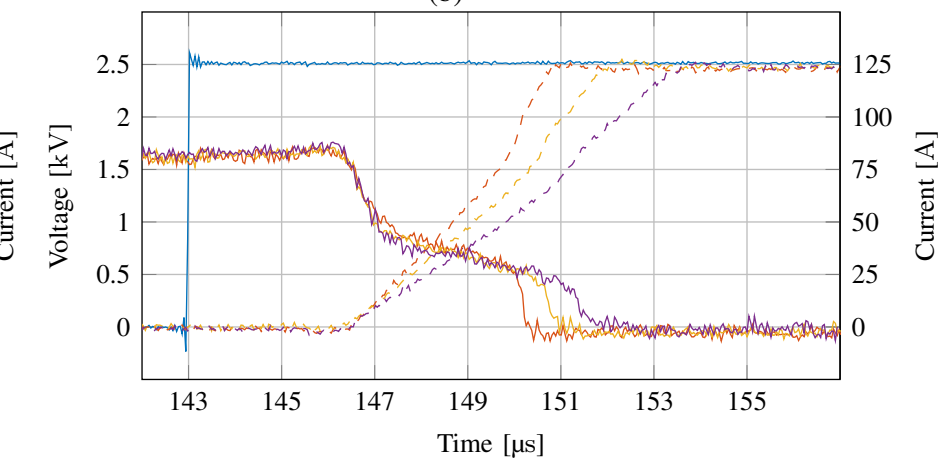

(d)

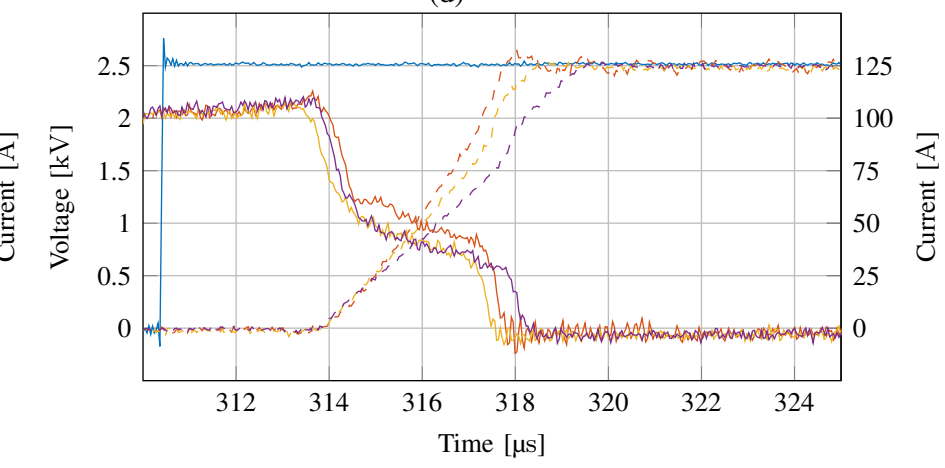

(f)

Fig. 7: Low current standard RC-IGCT turn-off with $R_{s}=5 \Omega$. From top to bottom, the turn-off current level is increased from $55 \mathrm{~A}$ to $75 \mathrm{~A}$ to $110 \mathrm{~A}$. Figs. (a), (c) and (e) refer to $S_{1}$, while Figs. (b), (d) and (f) refer to $S_{2}$. The switching pulse is shown in blue and using inverse logic in all of the subfigures. 


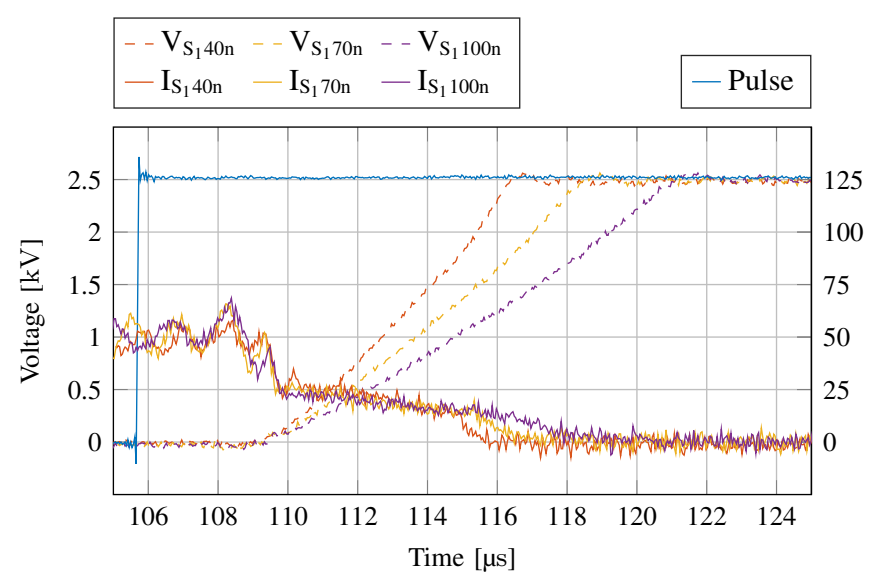

(a)

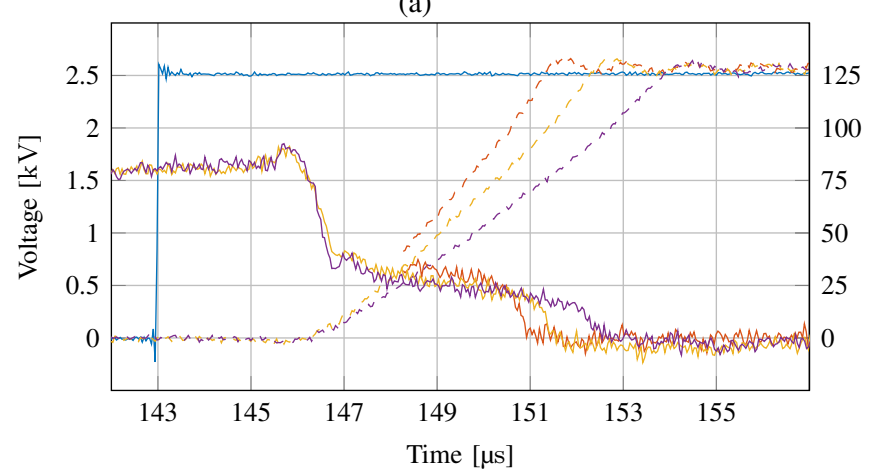

(c)

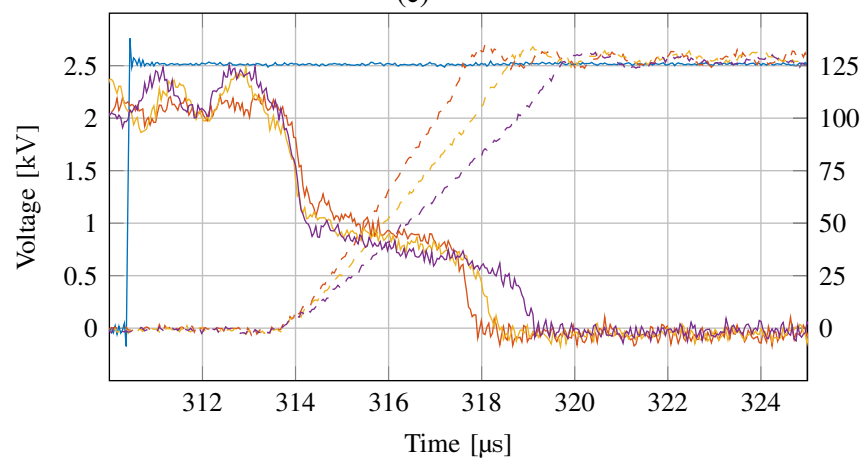

(e)

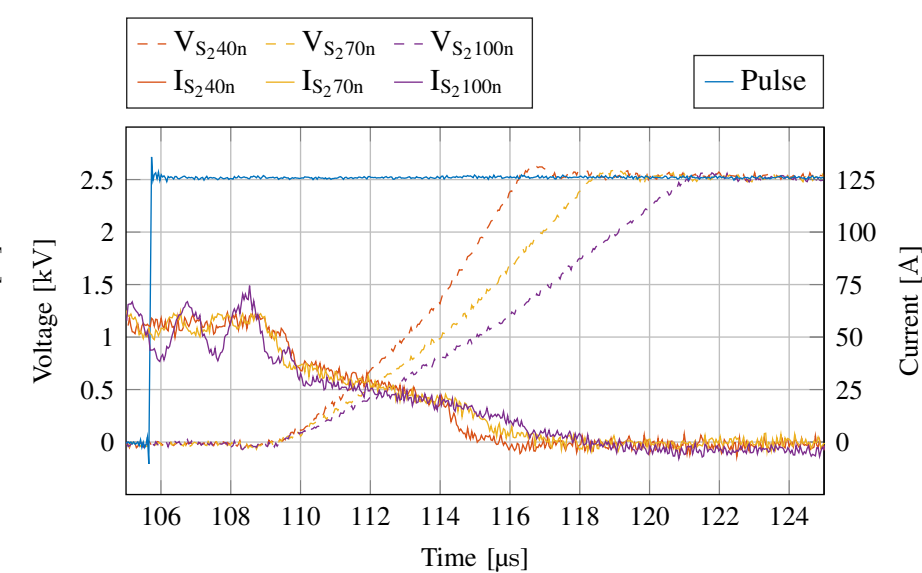

(b)

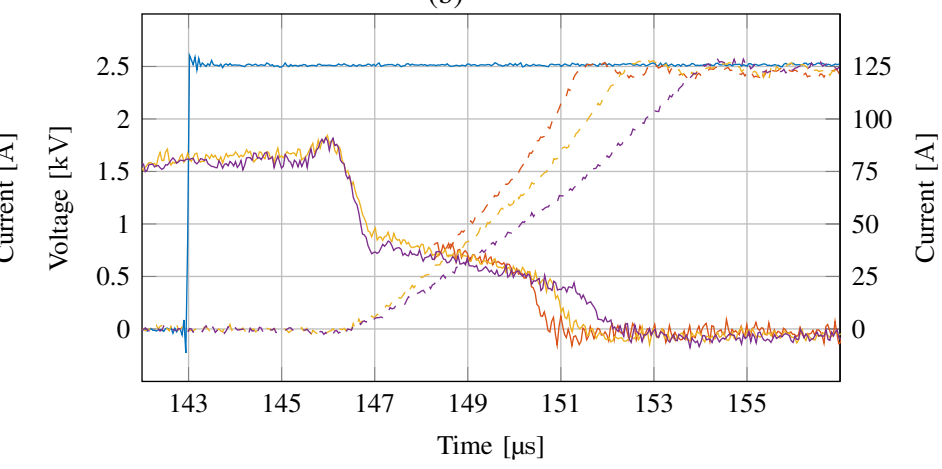

(d)

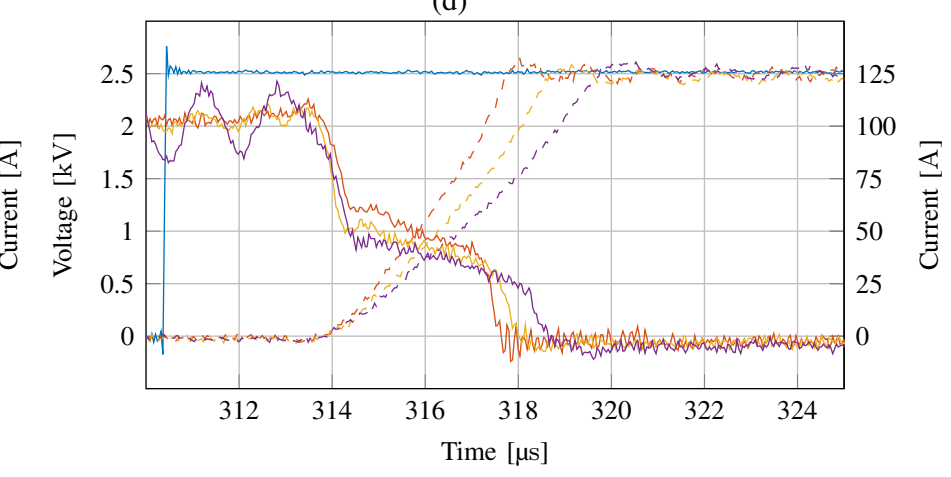

(f)

Fig. 8: Low current standard RC-IGCT turn-off with $R_{s}=0 \Omega$. From top to bottom, turn-off current level of $55 \mathrm{~A}$, $75 \mathrm{~A}$ and 110 A. Figs. (a), (c) and (e) refer to $S_{1}$, while Figs. (b), (d) and (f) refer to $S_{2}$. The turn-off with $R_{s}=0 \Omega$ is similar to $R_{s}=5 \Omega$, except for current oscillations due the turn-on.

voltage imbalances. This provides the important information that the difference in voltage between the two devices can be kept within approximately $10 \%$ of the device dc voltage, which is a relevant result considering this is the tolerance of the employed snubber capacitors. Third, in all the combinations of turn-off current and snubber capacitance, the voltage rise time remains well below $10 \mu \mathrm{s}$. This is another significant result since, as discussed, with an increased dc transformer switching frequency a fast transition between conducting and blocking state is necessary to maximise the portion of the period available for power transfer. Nevertheless it should be kept in mind that these results were obtained with devices at room temperature, as no infrastructure is in place to control the device case temperature. It is expected that an increase in junction temperature will result in a slower state transition requiring increased dead-time [37]. Finally, the energy dissipated at turn-off is always lower than was expected based on the linearisation of the device turn-off energy provided in the datasheet as a function of turn-off voltage and current. This is again a positive result, as it promises better than expected efficiency and reduced cooling effort of the devices. However, it should be noted that the calculated turn-off energy is only the energy dissipated in the IGCT itself, and not in the snubber. As different applications have different requirements in 

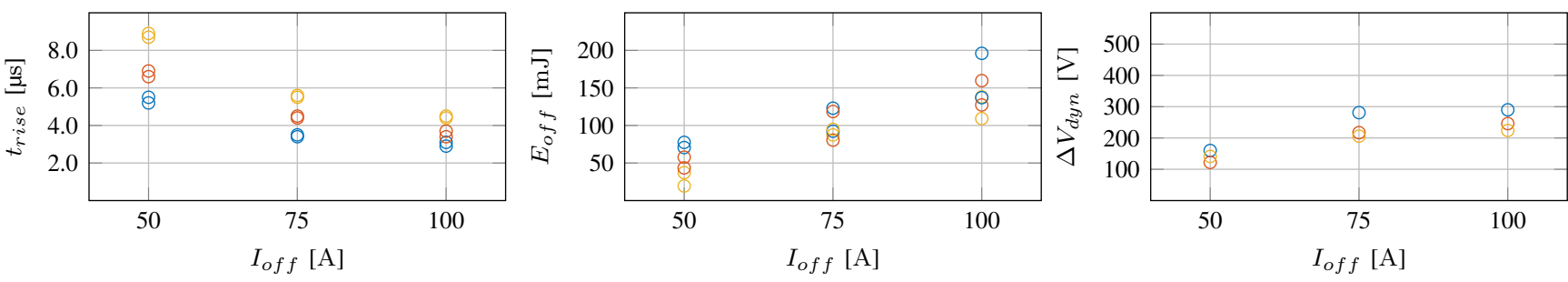

Fig. 9: Rise time, switching energy and $\Delta V_{d y n}$ during turn-off of standard RC-IGCTs as a function of $I_{o f f}$ for: $\circ C_{s}=40 \mathrm{nF}$, $\circ 70 \mathrm{nF}$, and $\odot 100 \mathrm{nF}$, respectively.

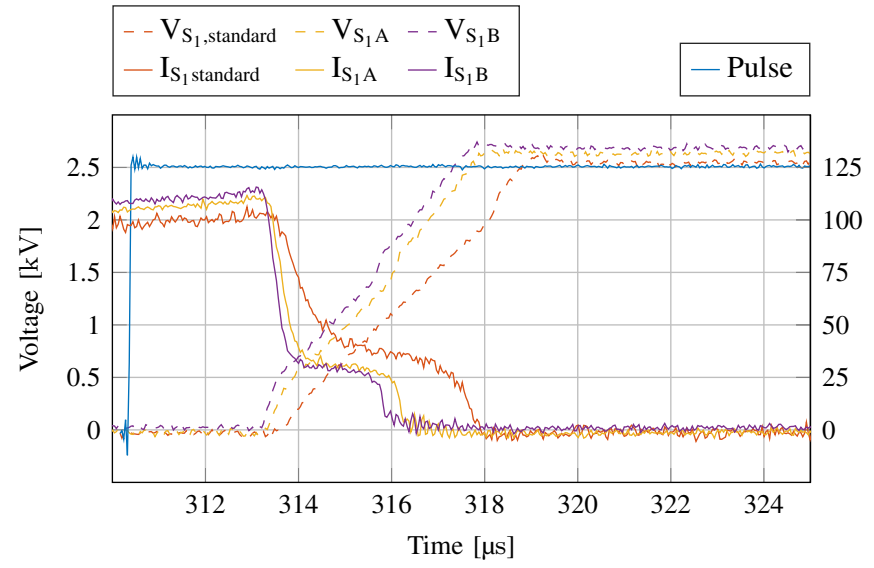

(a)

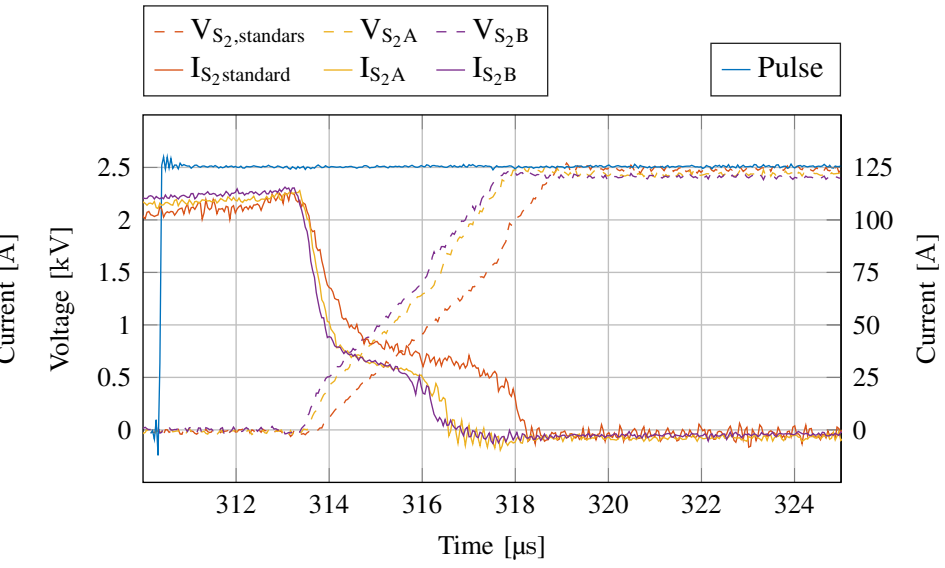

(b)

Fig. 10: Comparison of turn-off of (a) $S_{1}$ and (b) $S_{2}$ at $110 \mathrm{~A}$ for standard, irradiated $A$ and irradiated $B$ devices, with a $C_{s}=100 \mathrm{nF}$.

terms of admissible dead-time, turn-off energy, and dynamic voltage imbalance, it is not possible to provide a general recommendation for the optimal value of snubber capacitance under low current switching conditions. The obtained results show that a decrease in turn-off losses can be achieved either by a reduction in turn-off current, and by an increase in snubber capacitance. The same goes for improved dynamic voltage sharing. Nevertheless, based on the tests that have been carried out, a suggestion can be made as to which criteria to use with the goal of selecting an appropriate value of turn-off current and snubber capacitance in a given application. Once the designer has determined what is the maximum acceptable duration of the dead-time, several combinations of turn-off current and snubber capacitance can satisfy the condition. It is recommended that the designed select the combination with the lowest value of turn-off current, with a capacitance value that is still able to guarantee a dynamic voltage sharing between the series connected devices that is deemed to be acceptable in the application. This is because a decrease in turn-off current is found to be more beneficial than an increase in snubber capacitance both in therms of turn-off losses and in terms of voltage sharing. Note that this discussion is based solely on turn-off energy considerations, and not on the effect on device lifetime. Finally, it should be highlighted that the removal of the snubber capacitance is also not a feasible option, as testing which is not reported in the context of this paper has shown that in the absence of snubbers the voltage sharing between series connected devices is not sufficient for safe operation.

\section{B. Higher Electron Irradiated RC-IGCTs}

Having established the performance of standard IGCTs in low turn-off current and low snubber capacitance conditions as a benchmark, the performance of Irradiated $A$ and irradiated $B$ engineering samples can be tested and compared. The devices are made to perform the same tests as the commercial GCTs. An example of direct comparison of the switching waveforms is shown in Fig. 10, and the difference in performance is summarised in Fig. 11. This figure compares the devices in terms of turn-off energy, voltage rise times and peak dynamic voltage imbalance. For each value of snubber capacitance, one plot in Fig. 11 is presented. From left to right, values of $40 \mathrm{nF}$, $70 \mathrm{nF}$ and $100 \mathrm{nF}$ are used.

The first row of the figure, pertaining to turn-off energy, shows a clear distinction in behaviour between the commercial and irradiated $A$ and irradiated $B$ GCTs. The effect is more pronounced the lower the snubber capacitance value. For the $A$ and $B$ devices, no matter the turn-off current value, the turn-off energy never exceeds $100 \mathrm{~mJ}$ (except for one outlier point for $100 \mathrm{nF}$ snubber capacitance). In contrast, the turnoff energy of the standard GCTs reaches almost double this level end exhibits strong variations with turn-off current value, 


\section{IEEE TRANSACTIONS ON POWER ELECTRONICS}

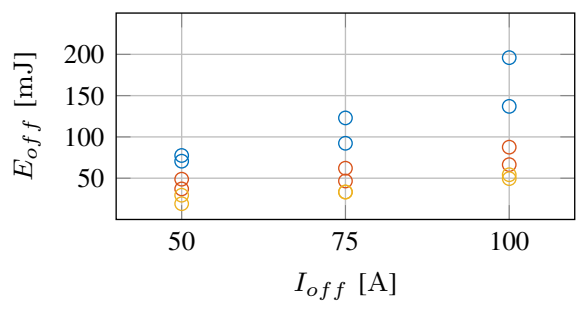

(a) $C_{s}=40 \mathrm{nF}$

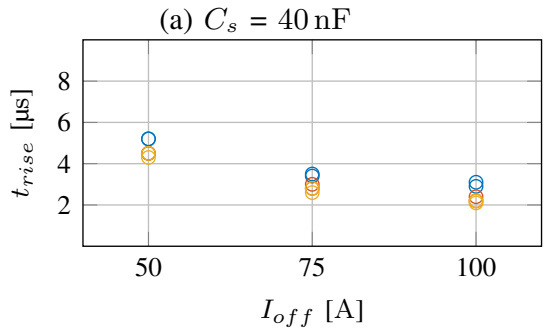

(d) $C_{s}=40 \mathrm{nF}$

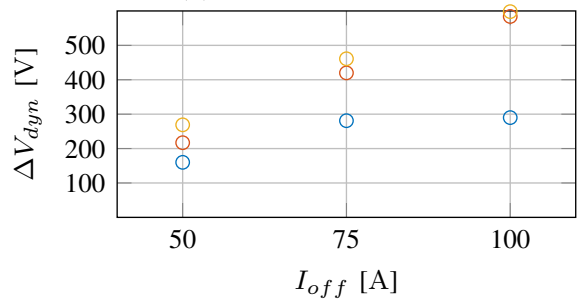

(g) $C_{s}=40 \mathrm{nF}$

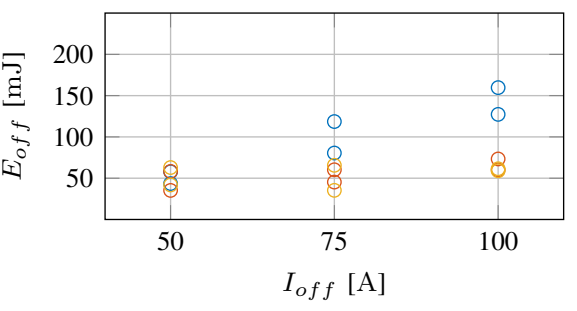

(b) $C_{s}=70 \mathrm{nF}$

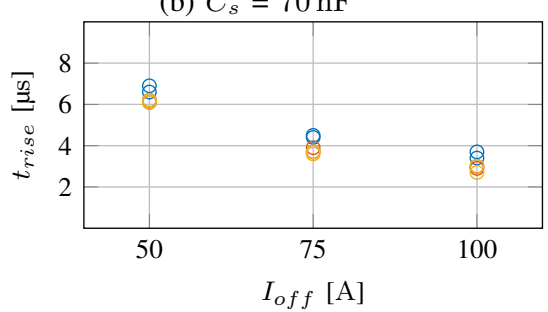

(e) $C_{s}=70 \mathrm{nF}$

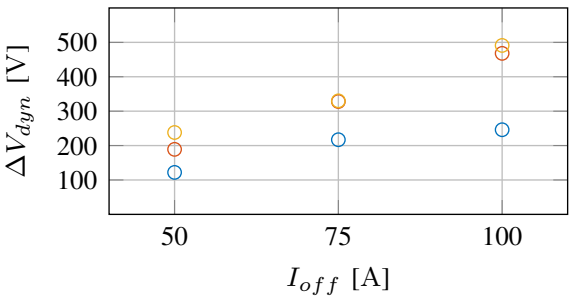

(h) $C_{s}=70 \mathrm{nF}$

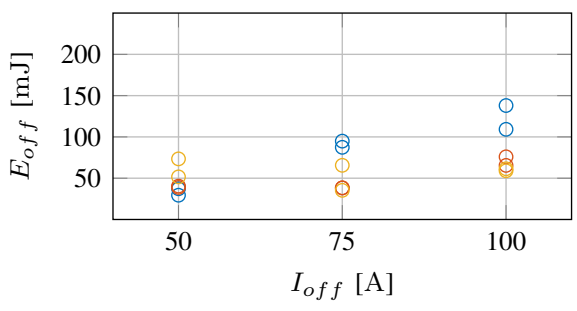

(c) $C_{s}=100 \mathrm{nF}$

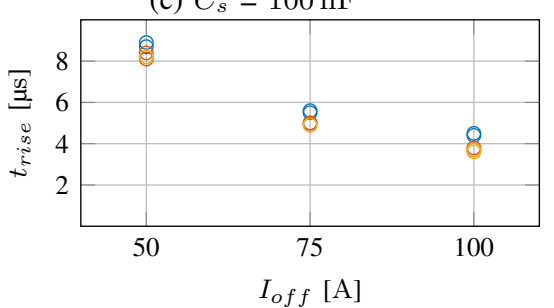

(f) $C_{s}=100 \mathrm{nF}$

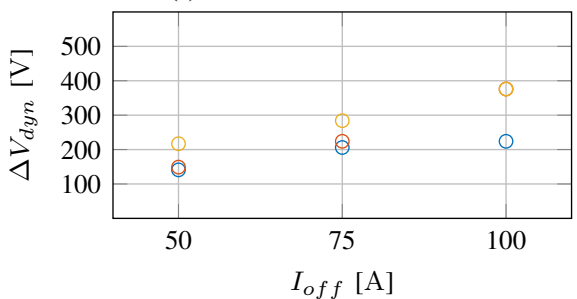

(i) $C_{s}=100 \mathrm{nF}$

Fig. 11: Comparison of switching energy (top), voltage rise time (center) and $\Delta V_{d y n}$ (bottom) during turn-off as a function of $I_{\text {off }}$. The employed GCTs are: $\bigcirc$ standard commercial devices, $\bigcirc$ irradiated $A$, and $\odot$ irradiated $B$.

ultimately due to the longer carrier lifetime and increased junction sweep-out time of the standard devices. One can also see by comparing the plots how an increase in snubber capacitance value, while it does not significantly affect the switching energy for irradiated devices, it does reduce the difference in switching energies between $S_{1}$ and $S_{2}$ for all tested GCTs. This is because as the dynamic voltage sharing improves, so does the sharing of the turn-off energy, since the current in the devices is the same due to their series connection.

The second row of Fig. 11 displays the trends in voltage rise time between the tested GCTs, which are as expected. The figure shows how the voltage rise time is a for the most part a function of turn-off current and snubber capacitance values. This is desired, as the purpose of the addition of snubbers in parallel to the devices is for the snubber to be the primary factor determining the GCT voltage sharing. It can be clearly seen how increased levels of turn-off current result in faster voltage rise, and also how increased snubber capacitance values result in a slower voltage rise. While standard GCTs are slightly slower than their irradiated counterparts across the board, this difference is small compared to that determined by snubber capacitances.

Finally, the last row of the figure displays the peak voltage differences between $S_{1}$ and $S_{2}$ during turn-off. Here, one can again see a difference in trends between the commercial and irradiated $A$ and irradiated $B$ devices. While the commercial devices exhibit a clear increase in voltage difference between a turn-off current of $50 \mathrm{~A}$ and $75 \mathrm{~A}$, this difference is proportionally much less significant when stepping up from a turnoff current of $75 \mathrm{~A}$ to $100 \mathrm{~A}$. This is not the case with $A$ and $B$ GCTs, which increase their peak voltage difference almost linearly with the increase of the turn-off current, likely due to the better turn-off delay matching of standard devices. On the other hand, all devices exhibit the desired reduction in voltage difference with the use of a larger snubber capacitance, with the irradiated devices reducing the peak voltage difference further than standard devices, especially at higher turn-off current levels. The characterisation of RC-IGCTs that underwent increased irradiation levels yields the expected results, and the comparison with standard commercial devices highlights a case for their employment in the the proposed SRC-LLC based DCX application. The devices provide a reduction in turn-off loss of up to $50 \%$ compared to standard devices, at the cost of an increased, but still safe, voltage unbalance. Ultimately, as the conduction losses of increased irradiation devices are also higher, the trade-off needs to be carefully evaluated and is the topic of future work.

The DP tests carried out in this paper are used to characterise only the low current turn-off behaviour of the IGCTs 


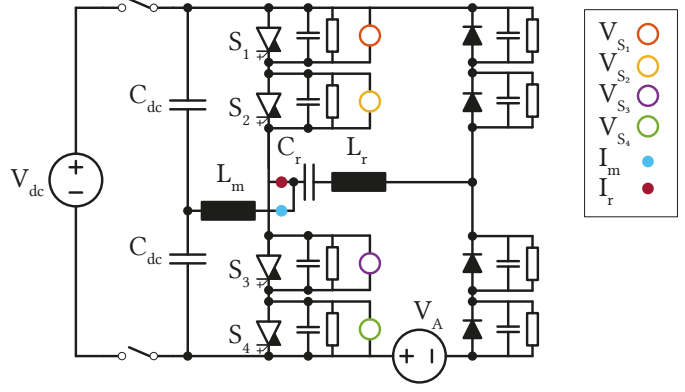

Fig. 12: Test setup configured for resonant operation. $C_{d c}$ $=1.3 \mathrm{mF}, L_{m}=6.3 \mathrm{mH}, C_{r}=85 \mu \mathrm{F}, L_{r}=5.5 \mu \mathrm{H}, f_{r}=$ $7.35 \mathrm{kHz}$.

under test. While the DP test would usually provide useful information concerning the device turn-on as well as turnoff, this is not the case in this context. This is because the conditions at turn-on witnessed by the devices in the DP test setup are not soft switching conditions. In the DP test setup, the DUTs are turned on with the full DC link voltage being applied to the terminals of the series connection of $S_{1}$ and $S_{2}$, and the current rate of increase is limited by the clamp circuit. This results in the snubbers in parallel with these two devices being charged, and the snubbers in parallel with $S_{3}$ and $S_{4}$ being completely discharged. At the time of turn-on, the snubbers in parallel with $S_{1}$ and $S_{2}$ quickly discharge into the device and the snubber resistor (if present), while the snubbers in parallel with $S_{3}$ and $S_{4}$ provide a current path bypassing $L_{m}$ and resulting in a large current spike limited only by the clamp inductor $L_{c l}$, as can be seen in Fig. 6c. This turn-on is not representative of turn-on that takes place in soft switching conditions. Soft turn-on would result for $S_{1}$ and $S_{2}$ in either ZVS or ZCS conditions, resulting in a virtually lossless commutation. It is for this reason that tests are also carried out also with no snubber resistor $\left(R_{s}=0 \Omega\right)$. While this would lead to high current peaks during turnon under hard switched conditions, under soft turn-on the snubbers are naturally discharged before the turn-on of the device, leading to lower losses and smaller snubbers. While their turn-on behaviour cannot be explored in this test setup, their turn-off was considered and results were presented. As the turn-off losses are expected to be the critical factor limiting the maximum switching frequency, their evaluation and the characterisation of the series connected low current IGCT turnoff was the main goal of this analysis.

\section{Resonant Operation}

Finally, the operation of series connected RC-IGCTs at a frequency of $5 \mathrm{kHz}$ is presented. The results displayed in this section are preliminary and no analysis of the effect of temperature on the presented waveforms is performed. The displayed waveforms refer to the devices' junction at ambient temperature (beginning of the test). For resonant operation, the test setup in Fig. 6a is reconfigured to imitate the operation of the LLC-SRC. Fig. 12 displays this, showing the addition of the resonant tank composed by $L_{r}$ and $C_{r}$, and the rectifier

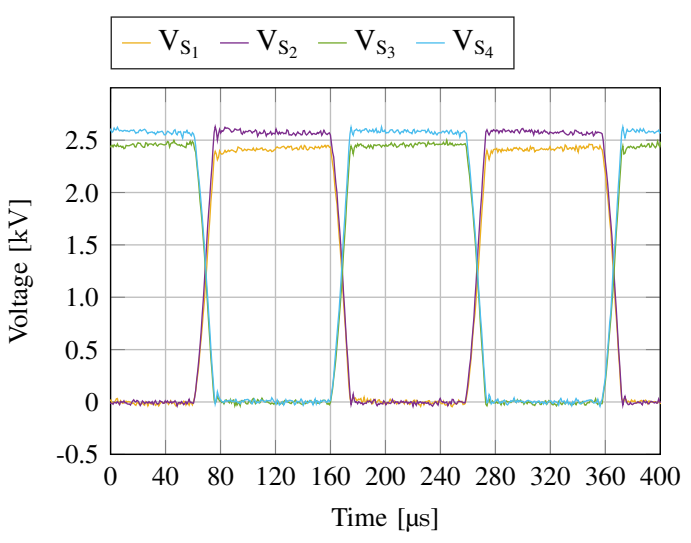

(a)

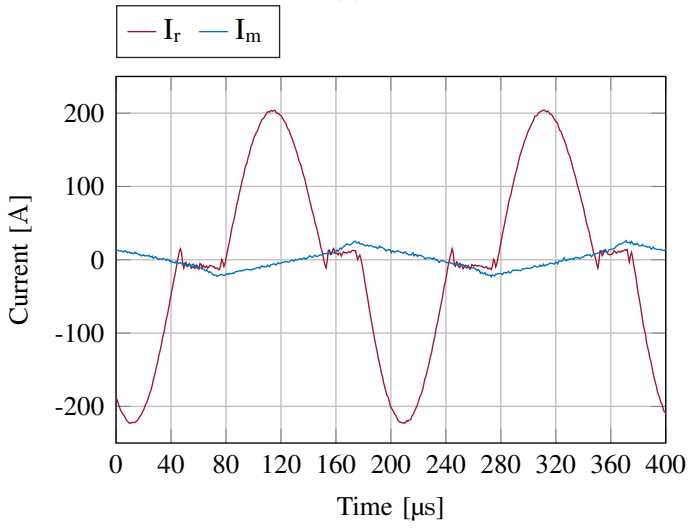

(b)

Fig. 13: $5 \mathrm{kHz}$ RC-IGCT series connected resonant operation (a) voltages and (b) currents.

diodes circulating power back to the DC link capacitors. $L_{m}$ conducts the equivalent of the transformer magnetising current $I_{m}$, while a second voltage source $V_{A}$ (MAGNA $20 \mathrm{~V}$, $2.2 \mathrm{kA}$ ) provides the simulated converter load resonant current $I_{r}$. This is achieved by $V_{A}$ simulating a voltage difference between the terminals of the rectifier and IGCTs. The setup is operated at its maximum circulated power, setting $V_{A}=$ 20 V. Fig. 13 displays the sensed voltages and currents. The waveforms displayed in the figure are obtained with a resonant frequency of the resonant tank equal to $7.35 \mathrm{kHz}$, which is significantly higher than the switching frequency of $5 \mathrm{kHz}$. This is a consequence of the relatively slow RC-IGCT turnoff and turn-on process, which imposes here a dead time of $20 \mu \mathrm{s}$, or $10 \%$ of the period. Below this values of dead time the IGCT turn-on takes place before the end of the turnoff of the complementary device, resulting in loss of ZVS. The snubber capacitance value used in resonant operation is of $20 \mathrm{nF}$, for both diodes and RC-IGCTs with the turn-off current having been further reduced to approximately $20 \mathrm{~A}$. In spite of this ultra-low turn-off current value, the devices are able to effectively turn $O F F$, and a power of almost $0.5 \mathrm{MW}$ was circulated in the setup. Higher power operation would be possible with increased voltage of $V_{A}$, but this is not possible with the available power supply. 
Very importantly, the test confirms that in these preliminary conditions, ZVS is maintained in resonant operation, validating the initial hypothesis of negligible device turn-on losses. Further analysis of the sources of power loss in this operation is required. Nevertheless, the presented resonant operation validates the feasibility of an SRC-based providing isolation through a medium frequency transformer.

\section{Conclusion}

To provide the technological foundation for the future development of IGCT-based DCX, this paper has demonstrated low current turn-off of series connected $4.5 \mathrm{kV}, 68 \mathrm{~mm}$ RC-IGCTs with multiple turn-off current levels and snubber capacitances. The performance of both commercial and purpose-optimised devices is evaluated and the improved turn-off performance of the latter is demonstrated. Trade-offs related to the increase switching performance are also discussed, in terms of switching energy and device voltage sharing. Finally, RC-IGCTs are operated in series connection at the frequency of $5 \mathrm{kHz}$ in conditions equivalent to those of an SRC-LLC converter. The presented results are promising for future research focusing on series connected RC-IGCTs operation in resonant dc-dc converter topologies.

\section{ACKNOWLEDGEMENTS}

The results presented in this paper are a part of the EMPOWER project that has received funding from the European Research Council (ERC) under the European Union's Horizon 2020 research and innovation programme (Grant agreement No. 818706).

\section{REFERENCES}

[1] M. S. Agamy, D. Dong, L. J. Garcés, Y. Zhang, M. E. Dame, X. Wu, and Y. Pan, "A high power medium voltage resonant dual active bridge for mvdc ship power networks," IEEE Journal of Emerging and Selected Topics in Power Electronics, vol. 5, no. 1, pp. 88-99, 2017.

[2] R. Soman, M. M. Steurer, T. A. Toshon, M. O. Faruque, and R. M. Cuzner, "Size and weight computation of mvdc power equipment in architectures developed using the smart ship systems design environment," IEEE Journal of Emerging and Selected Topics in Power Electronics, vol. 5, no. 1, pp. 40-50, 2017.

[3] G. L. Kusic, G. F. Reed, J. Svensson, and Z. Wang, "A case for medium voltage dc for distribution circuit applications," in 2011 IEEE/PES Power Systems Conference and Exposition, 2011, pp. 1-7.

[4] A. Hinz, M. Stieneker, and R. W. De Doncker, "Impact and opportunities of medium-voltage dc grids in urban railway systems," in 2016 18th European Conference on Power Electronics and Applications (EPE'16 ECCE Europe), 2016, pp. 1-10.

[5] S. Cui, N. Soltau, and R. W. De Doncker, "A high step-up ratio softswitching dedc converter for interconnection of mvdc and hvdc grids," IEEE Transactions on Power Electronics, vol. 33, no. 4, pp. 29863001, 2018.

[6] L. Zhang, K. Sun, Y. Xing, L. Feng, and H. Ge, "A modular gridconnected photovoltaic generation system based on dc bus," IEEE Transactions on Power Electronics, vol. 26, no. 2, pp. 523-531, 2011.

[7] S. Vighetti, J. Ferrieux, and Y. Lembeye, "Optimization and design of a cascaded dc/dc converter devoted to grid-connected photovoltaic systems," IEEE Transactions on Power Electronics, vol. 27, no. 4, pp. 2018-2027, 2012.

[8] V. M. Iyer, S. Gulur, G. Gohil, and S. Bhattacharya, "An approach towards extreme fast charging station power delivery for electric vehicles with partial power processing," IEEE Transactions on Industrial Electronics, vol. 67, no. 10, pp. 8076-8087, 2020.
[9] S. P. Engel, N. Soltau, H. Stagge, and R. W. De Doncker, "Dynamic and balanced control of three-phase high-power dual-active bridge dedc converters in dc-grid applications," IEEE Transactions on Power Electronics, vol. 28, no. 4, pp. 1880-1889, 2013.

[10] N. Soltau, D. Eggers, K. Hameyer, and R. W. De Doncker, "Iron losses in a medium-frequency transformer operated in a high-power dcdc converter," IEEE Transactions on Magnetics, vol. 50, no. 2, pp. 953956, 2014.

[11] J. Voss, S. P. Engel, and R. W. De Doncker, "Control method for avoiding transformer saturation in high-power three-phase dual-active bridge dcdc converters," IEEE Transactions on Power Electronics, vol. 35, no. 4, pp. 4332-4341, 2020.

[12] J. Hu, P. Joebges, G. C. Pasupuleti, N. R. Averous, and R. W. De Doncker, "A maximum-output-power-point-tracking-controlled dualactive bridge converter for photovoltaic energy integration into mvdc grids," IEEE Transactions on Energy Conversion, vol. 34, no. 1, pp. 170-180, 2019.

[13] D. Dujic, G. K. Steinke, M. Bellini, M. Rahimo, L. Storasta, and J. K. Steinke, "Characterization of $6.5 \mathrm{kv}$ igbts for high-power mediumfrequency soft-switched applications," IEEE Transactions on Power Electronics, vol. 29, no. 2, pp. 906-919, 2014.

[14] A. K. Tripathi, K. Mainali, D. C. Patel, A. Kadavelugu, S. Hazra, S. Bhattacharya, and K. Hatua, "Design considerations of a 15-kv sic igbtbased medium-voltage high-frequency isolated dcdc converter," IEEE Transactions on Industry Applications, vol. 51, no. 4, pp. 3284-3294, 2015.

[15] G. Ortiz, H. Uemura, D. Bortis, J. W. Kolar, and O. Apeldoorn, "Modeling of soft-switching losses of igbts in high-power highefficiency dual-active-bridge dc/dc converters," IEEE Transactions on Electron Devices, vol. 60, no. 2, pp. 587-597, 2013.

[16] T. Guillod, D. Rothmund, and J. W. Kolar, "Active magnetizing current splitting zvs modulation of a $7 \mathrm{kv} / 400 \mathrm{v}$ dc transformer," IEEE Transactions on Power Electronics, vol. 35, no. 2, pp. 1293-1305, 2020.

[17] J. Kucka and D. Dujic, "Smooth power direction transition of a bidirectional llc resonant converter for dc transformer applications," IEEE Transactions on Power Electronics, vol. 36, no. 6, pp. 6265-6275, 2021.

[18] P. Czyz, T. Guillod, F. Krismer, J. Huber, and J. W. Kolar, "Design and experimental analysis of $166 \mathrm{kw}$ medium-voltage medium-frequency air-core transformer for 1:1-dcx applications," IEEE Journal of Emerging and Selected Topics in Power Electronics, pp. 1-1, 2021.

[19] G. Ortiz, M. G. Leibl, J. E. Huber, and J. W. Kolar, "Design and experimental testing of a resonant dcdc converter for solid-state transformers," IEEE Transactions on Power Electronics, vol. 32, no. 10, pp. 7534-7542, 2017.

[20] S. Bernet, "Recent developments of high power converters for industry and traction applications," IEEE Transactions on Power Electronics, vol. 15 , no. 6, pp. 1102-1117, 2000.

[21] U. Vemulapati, M. Rahimo, M. Arnold, T. Wikström, J. Vobecky, B. Backlund, and T. Stiasny, "Recent advancements in igct technologies for high power electronics applications," in 2015 17th European Conference on Power Electronics and Applications (EPE'15 ECCEEurope), IEEE, 2015, pp. 1-10.

[22] H. F. Bilgin, M. Ermis, K. N. Kose, A. Cetin, I. Cadirci, A. Acik, T. Demirci, A. Terciyanli, C. Kocak, and M. Yorukoglu, "Reactive-power compensation of coal mining excavators by using a new-generation statcom," IEEE Transactions on Industry Applications, vol. 43, no. 1, pp. 97-110, 2007.

[23] Y. Suh and P. K. Steimer, "Application of igct in high-power rectifiers," IEEE Transactions on Industry Applications, vol. 45, no. 5, pp. 16281636, 2009.

[24] A. V. Rocha, H. de Paula, M. E. dos Santos, and B. J. Cardoso Filho, "A thermal management approach to fault-resilient design of three-level igct-based npc converters," IEEE Transactions on Industry Applications, vol. 49, no. 6, pp. 2684-2691, 2013.

[25] D. Andler, R. Álvarez, S. Bernet, and J. Rodríguez, "Experimental investigation of the commutations of a 31-anpc phase leg using 4.5kv5.5-ka igcts," IEEE Transactions on Industrial Electronics, vol. 60, no. 11 , pp. $4820-4830,2013$. 
[26] F. Filsecker, R. Alvarez, and S. Bernet, "Comparison of 4.5-kv presspack igbts and igcts for medium-voltage converters," IEEE Transactions on Industrial Electronics, vol. 60, no. 2, pp. 440-449, 2013.

[27] B. Ødegård, D. Weiss, T. Wikström, and R. Baumann, "Rugged mmc converter cell for high power applications," in 2016 18th European Conference on Power Electronics and Applications (EPE'16 ECCE Europe), IEEE, 2016, pp. 1-10.

[28] D. Weiss, M. Vasiladiotis, C. Banceanu, N. Drack, B. Odegard, and A. Grondona, "Igct based modular multilevel converter for an ac-ac rail power supply," in PCIM Europe 2017; International Exhibition and Conference for Power Electronics, Intelligent Motion, Renewable Energy and Energy Management, VDE, 2017, pp. 1-8.

[29] D. Stamenkovic and D. Dujic, "Application of the igct for resonant conversion with low switching losses," IEEJ Journal of Industry Applications, vol. 9, no. 3, pp. 244-253, 2020.

[30] D. Stamenkovic, U. R. Vemulapati, T. Stiasny, M. Rahimo, and D. Duji, "Igct low-current switchingtcad and experimental characterization," IEEE Transactions on Industrial Electronics, vol. 67, no. 8, pp. 63026311, 2020.

[31] D. Stamenkovic, "Igct based solid state resonant conversion," EPFL, Tech. Rep., 2020.

[32] Qingguang $\mathrm{Yu}$, Lili Li, and Qirong Jiang, "High power electronic device measurements of four igcts (4.5 kv/4.0 ka) in series connection," in Fifth World Congress on Intelligent Control and Automation (IEEE Cat. No.04EX788), vol. 5, 2004, 4446-4449 Vol.5.

[33] S. Bhattacharya, "Series connected igct based high power three-level neutral point clamped voltage source inverter pole for facts applications," in 2005 IEEE 36th Power Electronics Specialists Conference, 2005, pp. 2315-2321.

[34] A. Kerim, J. Steinke, B. Oedegard, and S. Reichert, "Measurements on igcts and diodes series connection," in Proceedings of the 2011 14th European Conference on Power Electronics and Applications, 2011, pp. $1-10$

[35] A. Nagel, S. Bernet, P. K. Steimer, and O. Apeldoorn, "A 24 mva inverter using igct series connection for medium voltage applications," in Conference Record of the 2001 IEEE Industry Applications Conference. 36th IAS Annual Meeting (Cat. No.01CH37248), vol. 2, 2001, 867-870 vol.2.

[36] Lu Jiming, Wang Dan, Mao Chengxiong, and Fan Shu, "Study of rcsnubber for series igcts," in Proceedings. International Conference on Power System Technology, vol. 1, 2002, 595-599 vol.1.

[37] A. Nagel, S. Bernet, T. Bruckner, P. K. Steimer, and O. Apeldoorn, "Characterization of igcts for series connected operation," in Conference Record of the 2000 IEEE Industry Applications Conference. Thirty-Fifth IAS Annual Meeting and World Conference on Industrial Applications of Electrical Energy (Cat. No.00CH37129), vol. 3, 2000, 1923-1929 vol.3.

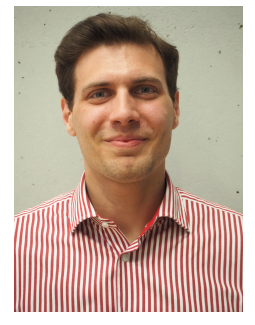

Gabriele Ulissi (S18) received the B.Sc. degree in electrical engineering from the Polytechnic of Turin, Italy, in 2016 and M.Sc. degree in electrical engineering from the École Polytechnique Fédérale de Lausanne (EPFL), Switzerland, in 2018. Since 2018, he is a Doctoral Assistant with the Power Electronics Laboratory at École Polytechnique Fédérale de Lausanne (EPFL), Switzerland. His research focuses on semiconductor-based protection of dc systems and dc transformers.

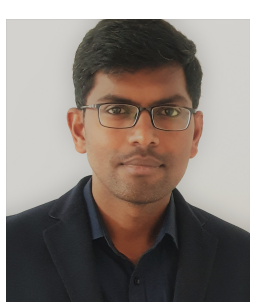

Umamaheswara Reddy Vemulapati (M12) received the bachelors degree in electrical engineering from RGMCET affiliated to JNTU Hyderabad, India. He received the Masters degree and the Ph.D. degree in electrical engineering from the University of Bremen, Germany. He joined ABB Switzerland Ltd., Corporate Research in 2011. Since then he worked on high-power semiconductor devices, such as IGCTs, IGBTs and Fast Recovery Diodes. Currently he is a ductors, Switzerland. principal engineer at Hitachi Energy, Semicon-

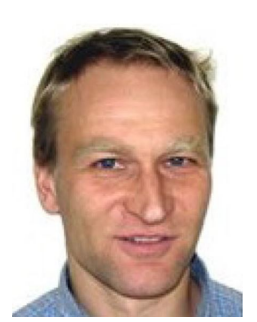

Thomas Stiasny received the Diploma and Ph.D. degrees in physics from FriedrichAlexander University, Erlangen, Germany, in 1991 and 1996, respectively. He joined ABB Semiconductors, Lenzburg, Switzerland in 1997. Areas of work were development of Bipolar semiconductors and reliability aspects of those. Currently he is a senior principal engineer at Hitachi Energy, Semiconductors, Switzerland.

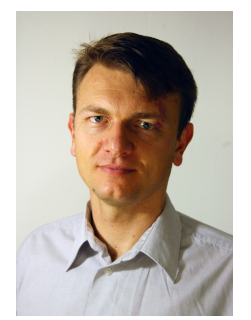

Drazen Dujic (S03-M09-SM12) received the Dipl.-Ing. and M.Sc. degrees from the University of Novi Sad, Novi Sad, Serbia, in 2002 and 2005, respectively, and the Ph.D. degree from Liverpool John Moores University, Liverpool, U.K., in 2008, all in electrical engineering. From 2002 to 2006, he was with the Department of Electrical Engineering, University of Novi Sad, as a Research Assistant. From 2006 to 2009, he was with Liverpool John Moores University, as a Research Associate. From 2009 to 2013, he was with the ABB Corporate Research Centre, Switzerland, as the Principal Scientist, working on the power electronics projects spanning the range from low-voltage/power SMPS in below kilowatt range to medium voltage high-power converters in a megawatt range. From 2010 to 2011, he was a member of a project team responsible for the development of the worlds first power electronic traction transformer successfully commissioned on the locomotive. From 2013 to 2014, he was with ABB Medium Voltage Drives, Turgi, Switzerland, as a Research and Development Platform Manager, responsible for ABBs largest IGCT-based medium voltage drive ACS6000. He is currently with the École Polytechnique Fédérale de Lausanne (EPFL), Lausanne, Switzerland, as an Associate Professor and the Director of the Power Electronics Laboratory. He has authored or coauthored more than 200 scientific publications and has led 18 patents. His current research interests include the areas of design and control of advanced high-power electronics systems for medium voltage applications. He has received the First Prize Paper Award from the Electric Machines Committee of the IEEE Industrial Electronics Society, in 2007. In 2014, he has received the Isao Takahashi Power Electronics Award for outstanding achievement in power electronics, and in 2018, the EPE Outstanding Service Award from the European Power Electronics and Drives Association. $\mathrm{He}$ is an Associate Editor of the IEEE TRANSACTIONS ON INDUSTRIAL ELECTRONICS, the IEEE TRANSACTIONS ON POWER ELECTRONICS, and the IET Electric Power Applications. 